\title{
VALENCIA: A nearest centroid classification method for vaginal microbial communities
}

\author{
M. T. France \\ B. Ma \\ P. Gajer \\ S. Brown \\ M. S. Humphrys \\ J. B. Holm \\ L. E. Waetjen \\ R. M. Brotman \\ J. Ravel
}

\section{Video Byte}

Keywords: Microbiome, centroid, VALENCIA, Taxonomic profiles, vaginal microbial communities, Community State Types, tool, analysis, microbial composition

Posted Date: February 25th, 2021

DOI: https://doi.org/10.21203/rs.3.rs-275922/v1

License: (9) (1) This work is licensed under a Creative Commons Attribution 4.0 International License. Read Full License 


\section{Abstract}

The human body contains a diverse array of microbial communities. Categorizing these communities can help microbiologists study how they influence human health. Although hierarchical clustering is the most common approach, newer techniques have been developed to assign more stable classifications. A recent study instead used a centroid classification algorithm to reproducibly place microbial communities into categories based on composition and structure. The researchers implemented their algorithm to assign community profiles to vaginal microbes. Using publicly available datasets containing vaginal samples from a variety of women, they were able to assign vaginal microbes to community state types (CSTs), which refer to the relative abundance of all phylotypes in an individual. Their work resulted in the development of a tool named VALENCIA, which classifies samples based on their similarity to a set of reference centroids. While work is ongoing to expand the reference datasets beyond North American samples. VALENCIA provides a much-needed solution for the robust and reproducible assignment of vaginal CSTs. allowing for unbiased analysis and comparison of a variety of microbial datasets. 